\title{
HADAMARD PRODUCT OF CERTAIN MEROMORPHIC STARLIKE AND CONVEX FUNCTIONS
}

\author{
M.L. MOGRA
}

\begin{abstract}
The author establishes certain results concerning the Hadamard product of meromorphic starlike and meromorphic convex functions analogous to those obtained by Vinod Kumar (J. Math. Anal. Appl. 113(1986), 230-234).
\end{abstract}

\section{Introduction}

Throughout the paper, let the functions of the form

$$
\phi(z)=c_{1} z-\sum_{n=2}^{\infty} c_{n} z^{n} \quad\left(c_{1}>0, c_{n} \geq 0\right)
$$

and

$$
\psi(z)=d_{1} z-\sum_{n=2}^{\infty} d_{n} z^{n} \quad\left(d_{1}>0, d_{n} \geq 0\right)
$$

be regular and univalent in the unit disc $U=\{z:|z|<1\}$; and let

$$
\begin{array}{ll}
f(z)=\frac{a_{0}}{z}+\sum_{n=1}^{\infty} a_{n} z^{n} \quad\left(a_{0}>0, a_{n} \geq 0\right), \\
f_{i}(z)=\frac{a_{0, i}}{z}+\sum_{n=1}^{\infty} a_{n, i} z^{n} \quad\left(a_{0, i}>0, a_{n, i} \geq 0\right), \\
g(z)=\frac{b_{0}}{z}+\sum_{n=1}^{\infty} b_{n} z^{n} \quad\left(b_{0}>0, b_{n} \geq 0\right),
\end{array}
$$

Received January 8, 1993.

1991 Mathematics Subject Classification. 30C45.

Key words and phrases. Hadamard product, meromorphic starlike functions, meromorphic convex functions, positive coefficients. 
and.

$$
g_{j}(z)=\frac{b_{0, j}}{z}+\sum_{n=1}^{\infty} b_{n, j} z^{n} \quad\left(b_{0, j}>0, b_{n, j} \geq 0\right)
$$

be regular and univalent in the punctured disc $D=\{z: 0<|z|<1\}$.

Let $S_{0}^{*}(\alpha, \beta)$ denote the class of functions $\phi(z)$ which satisfy the condition

$$
\left|z \phi^{\prime}(z) / \phi(z)-1\right|<\beta\left|z \phi^{\prime}(z) / \phi(z)+1-2 \alpha\right|
$$

for some $\alpha, \beta(0 \leq \alpha<1,0<\beta \leq 1)$ and for all $z \in U$; and let $C_{0}^{*}(\alpha, \beta)$ be the class of functions $\phi(z)$ for which $z \phi^{\prime}(z) \in S_{0}^{*}(\alpha, \beta)$. It is well known that the functions in $S_{0}^{*}(\alpha, \beta)$ and $C_{0}^{*}(\alpha, \beta)$ are respectively starlike and convex of order $\alpha$ and type $\beta$ with negative coefficients in $U$.

Denote by $\sum S_{0}^{*}(\alpha, \beta)$, the class of functions $f(z)$ which satisfy the condition

$$
\left|z f^{\prime}(z) / f(z)+1\right|<\beta\left|z f^{\prime}(z) / f(z)+2 \alpha-1\right|
$$

for some $\alpha, \beta(0 \leq \alpha<1,0<\beta \leq 1)$ and for all $z \in D$; and let $\sum C_{0}^{*}(\alpha, \beta)$ be the class of functions $f(z)$ for which $z f^{\prime}(z) \in \sum S_{0}^{*}(\alpha, \beta)$. The functions in $\sum S_{0}^{*}(\alpha, \beta)$ and $\sum C_{0}^{*}(\alpha, \beta)$ are respectively called meromorphic starlike and meromorphic convex of order $\alpha$ and type $\beta$ with positive coefficients in $D$. The class $\sum S_{0}^{*}(\alpha, \beta)$ with $a_{0}=1$ has extensively been studied in [4].

Using similar arguments as given in [4], we can easily prove the following results for functions in $\sum S_{0}^{*}(\alpha, \beta)$ and $\sum C_{0}^{*}(\alpha, \beta)$.

A function $f(z) \in \sum S_{0}^{*}(\alpha, \beta)$ if and only if

$$
\sum_{n=1}^{\infty}\left[\{n+1+\beta(n+2 \alpha-1)\} a_{n}\right] \leq 2 \beta(1-\alpha) a_{0}
$$

and $f(z) \in \sum C_{0}^{*}(\alpha, \beta)$ if and only if

$$
\sum_{n=1}^{\infty}\left[n\{n+1+\beta(n+2 \alpha-1)\} a_{n}\right] \leq 2 \beta(1-\alpha) a_{0} .
$$

The quasi-Hadamard product of two or more functions has recently been defined and used by Owa $[5,6,7]$, Kumar $[1,2,3]$, and others. Accordingly, the quasi-Hadamard product of two functions $\phi(z)$ and $\psi(z)$ is defined by

$$
\phi * \psi(z)=c_{1} d_{1} z-\sum_{n=2}^{\infty} c_{n} d_{n} z^{n}
$$

Let us define the Hadamard product of two meromorphic univalent functions $f(z)$ and $g(z)$ by

$$
f * g(z)=\frac{a_{0} b_{0}}{z}+\sum_{n=1}^{\infty} a_{n} b_{n} z^{n}
$$


The Hadamard product of more than two meromorphic univalent functions can similarly be defined.

In [2], Kumar obtained certain results concerning the quasi-Hadamard product of two or more functions in $S_{0}^{*}(\alpha, \beta)$ and $C_{0}^{*}(\alpha, \beta)$, and has shown that his results improve the corresponding results of Owa [7].

Since to a certain extent the work in the meromorphic univalent case has paralleled that of regular univalent case, one is tempted to search results analogous to Kumar [2] for meromorphic univalent functions in $D$. Thus we introduce the following class of meromorphic univalent functions in $D$.

A function $f(z) \in \sum_{k}^{*}(\alpha, \beta)$ if and only if

$$
\sum_{n=1}^{\infty}\left[n^{k}\{n+1+\beta(n+2 \alpha-1)\} a_{n}\right] \leq 2 \beta(1-\alpha) a_{0}
$$

where $0 \leq \alpha<1,0<\beta \leq 1$ and $k$ is any fixed nonnegative real number.

Evidently, $\sum_{0}^{*}(\alpha, \beta) \equiv \sum S_{0}^{*}(\alpha, \beta)$ and $\sum_{1}^{*}(\alpha, \beta) \equiv \sum C_{0}^{*}(\alpha, \beta)$. Further, $\sum_{k}^{*}(\alpha, \beta)$ $\subset \sum_{h}^{*}(\alpha, \beta)$ if $k>h \geq 0$, the containment being proper. Moreover, for any positive integer $k$, we have the following inclusive relation.

$$
\sum_{k}^{*}(\alpha, \beta) \subset \sum_{k-1}^{*}(\alpha, \beta) \subset \cdots \subset \sum_{2}^{*}(\alpha, \beta) \subset \sum C_{0}^{*}(\alpha, \beta) \subset \sum S_{0}^{*}(\alpha, \beta) .
$$

We also note that for every nonnegative real number $k$, the class $\sum_{k}^{*}(\alpha, \beta)$ is nonempty as the functions of the form

$$
f(z)=\frac{a_{0}}{z}+\sum_{n=1}^{\infty} n^{-k}\left\{\frac{2 \beta(1-\alpha)}{n+1+\beta(n+2 \alpha-1)}\right\} a_{0} \lambda_{n} z^{n}
$$

where $a_{0}>0, \lambda_{n} \geq 0$ and $\sum_{n=1}^{\infty} \lambda_{n} \leq 1$, satisfy the inequality (1).

The aim of the present paper is to establish certain results concerning the Hadamard product of meromorphic starlike and convex functions analogous to those obtained by Kumar [2]. It is interesting to note that our results are valid for the usual "Hadamard product" while Kumar [2] used in his results "quasi-Hadamard product" instead of the usual "Hadamard product".

\section{The Main Theorems}

Theorem 1. Let the functions $f_{i}(z)$ belong to the class $\sum C_{0}^{*}(\alpha, \beta)$ for every $i=1,2, \cdots, m$. Then the Hadamard product $f_{1} * f_{2} * \cdots * f_{m}(z)$ belongs to the class $\sum_{2 m-1}^{*}(\alpha, \beta)$.

Proof. it is sufficient to show that

$$
\sum_{n=1}^{\infty}\left[n^{2 m-1}\{n+1+\beta(n+2 \alpha-1)\} \prod_{i=1}^{m} a_{n, i}\right] \leq 2 \beta(1-\alpha)\left[\prod_{i=1}^{m} a_{0, i}\right] .
$$


Since $f_{i}(z) \in \sum C_{0}^{*}(\alpha, \beta)$, we have

$$
\sum_{n=1}^{\infty}\left[n\{n+1+\beta(n+2 \alpha-1)\} a_{n, i}\right] \leq 2 \beta(1-\alpha) a_{0, i}
$$

for every $i=1,2, \cdots, m$. Therefore,

$$
n\{n+1+\beta(n+2 \alpha-1)\} a_{n, i} \leq 2 \beta(1-\alpha) a_{0, i}
$$

or

$$
a_{n, i} \leq\left[\frac{2 \beta(1-\alpha)}{n\{n+1+\beta(n+2 \alpha-1)\}}\right] a_{0, i},
$$

for every $i=1,2, \cdots, m$. The right-hand expression of the last inequality is not greater than $n^{-2} a_{0, i}$. Hence

$$
a_{n, i} \leq n^{-2} a_{0, i}
$$

for every $i=1,2, \cdots, m$.

Using (3) for $i=1,2, \cdots, m-1$, and (2) for $i=m$, we obtain

$$
\begin{aligned}
& \sum_{n=1}^{\infty}\left[n^{2 m-1}\{n+1+\beta(n+2 \alpha-1)\} \prod_{i=1}^{m} a_{n, i}\right] \\
\leq & \sum_{n=1}^{\infty}\left[n^{2 m-1}\{n+1+\beta(n+2 \alpha-1)\}\left(n^{-2(m-1)} \prod_{i=1}^{m-1} a_{0, i}\right) a_{n, m}\right] \\
= & {\left[\prod_{i=1}^{m-1} a_{0, i}\right] \sum_{n=1}^{\infty}\left[n\{n+1+\beta(n+2 \alpha-1)\} a_{n, m}\right] } \\
\leq & 2 \beta(1-\alpha)\left[\prod_{i=1}^{m} a_{0, i}\right] .
\end{aligned}
$$

Hence $f_{1} * f_{2} * \cdots * f_{m}(z) \in \sum_{2 m-1}^{*}(\alpha, \beta)$.

Theorem 2. Let the functions $f_{i}(z)$ belong to the class $\sum S_{0}^{*}(\alpha, \beta)$ for every $i=1,2, \cdots, m$. Then the Hadamard product $f_{1} * f_{2} * \cdots * f_{m}(z)$ belongs to the class $\sum_{m-1}^{*}(\alpha, \beta)$.

Proof. Since $f_{i}(z) \in \sum S_{0}^{*}(\alpha, \beta)$, we have

$$
\sum_{n=1}^{\infty}\left[\{n+1+\beta(n+2 \alpha-1)\} a_{n, i}\right] \leq 2 \beta(1-\alpha) a_{0, i}
$$

for every $i=1,2, \cdots, m$. Therefore

$$
a_{n, i} \leq\left[\frac{2 \beta(1-\alpha)}{\{n+1+\beta(n+2 \alpha-1)\}}\right] a_{0, i}
$$


and hence

$$
a_{n, i} \leq n^{-1} a_{0, i}
$$

for every $i=1,2, \cdots, m$.

Using [5] for $i=1,2, \cdots, m-1$, and [4] for $i=m$, we get

$$
\begin{aligned}
& \sum_{n=1}^{\infty}\left[n^{m-1}\{n+1+\beta(n+2 \alpha-1)\} \prod_{i=1}^{m} a_{n, i}\right] \\
\leq & \sum_{n=1}^{\infty}\left[n^{m-1}\{n+1+\beta(n+2 \alpha-1)\}\left(n^{-(m-1)} \prod_{i=1}^{m-1} a_{0, i}\right) a_{n, m}\right] \\
= & {\left[\prod_{i=1}^{m-1} a_{0, i}\right] \sum_{n=1}^{\infty}\left[\{n+1+\beta(n+2 \alpha-1)\} a_{n, m}\right] } \\
\leq & 2 \beta(1-\alpha)\left[\prod_{i=1}^{m} a_{0, i}\right] .
\end{aligned}
$$

Hence $f_{1} * f_{2} * \cdots * f_{m}(z) \in \sum_{m-1}^{*}(\alpha, \beta)$.

Theorem 3. Let the functions $f_{i}(z)$ belong to the class $\sum C_{0}^{*}(\alpha, \beta)$ for every $i=1,2, \cdots, m$; and let the functions $g_{j}(z)$ belong to the class $\sum S_{0}^{*}(\alpha, \beta)$ for every $j=1,2, \cdots, q$. Then the Hadamard product $f_{1} * f_{2} * \cdots * f_{m} * g_{1} * g_{2} * \cdots * g_{q}(z)$ belongs to the class $\sum_{2 m+q-1}^{*}(\alpha, \beta)$.

Proof. It is sufficient to show that

$$
\begin{aligned}
& \sum_{n=1}^{\infty}\left[n^{2 m+q-1}\{n+1+\beta(n+2 \alpha-1)\}\left(\prod_{i=1}^{m} a_{n, i} \prod_{j=1}^{q} b_{n, j}\right)\right] \\
& \leq 2 \beta(1-\alpha)\left(\prod_{i=1}^{m} a_{0, i} \prod_{j=1}^{q} b_{0, j}\right) .
\end{aligned}
$$

Since $f_{i}(z) \in \sum C_{0}^{*}(\alpha, \beta)$, the inequalities [2] and [3] hold for every $i=1,2, \cdots, m$. Further, since $g_{j}(z) \in \sum S_{0}^{*}(\alpha, \beta)$, we have

$$
\sum_{n=1}^{\infty}\left[\{n+1+\beta(n+2 \alpha-1)\} b_{n, j}\right] \leq 2 \beta(1-\alpha) b_{0, j}
$$

for every $j=1,2, \cdots, q$. Whence we obtain

$$
b_{n, j} \leq n^{-1} b_{0, j}
$$

for every $j=1,2, \cdots, q$ 
Using [3] for $i=1,2, \cdots, m$; [7] for $j=1,2, \cdots, q-1$; and [6] for $j=q$, we get

$$
\begin{aligned}
& \sum_{n=1}^{\infty}\left[n^{2 m+q-1}\{n+1+\beta(n+2 \alpha-1)\}\left(\prod_{i=1}^{m} a_{n, i} \prod_{j=1}^{q} b_{n, j}\right)\right] \\
\leq & \sum_{n=1}^{\infty}\left[n^{2 m+q-1}\{n+1+\beta(n+2 \alpha-1)\}\left(n^{-2 m} n^{-(q-1)} \prod_{i=1}^{m} a_{0, i} \prod_{j=1}^{q-1} b_{0, j}\right) b_{n, q}\right] \\
= & \left(\prod_{i=1}^{m} a_{0, i} \prod_{j=1}^{q-1} b_{0, j}\right) \sum_{n=1}^{\infty}\left[\{n+1+\beta(n+2 \alpha-1)\} b_{n, q}\right] \\
\leq & 2 \beta(1-\alpha)\left(\prod_{i=1}^{m} a_{0, i} \prod_{j=1}^{q} b_{0, j}\right)
\end{aligned}
$$

Hence $f_{1} * f_{2} * \cdots * f_{m} * g_{1} * g_{2} * \cdots * g_{q} \in \sum_{2 m+q-1}^{*}(\alpha, \beta)$.

We note that the required estimate can also be obtained by using [3] for $i=$ $1,2, \cdots, m-1$; [7] for $j=1,2, \cdots, q$; and [2] for $i=m$.

\section{References}

[1] V. Kumar, "Hadamard product of certain starlike functions," J. Math. Anol. Appl. 110(1985), 425-428.

[2] V. Kumar, "Hadamard product of certain starlike functions II" J. Math. Anal. Appl. 113(1986), 230-234.

[3] V. Kumar, "Quasi-Hadamard product of certain univalent functions," J. Math. Anal. Appl. 126 (1987), 70-77.

[4] M. L. Mogra. T. R. Reddy and O. P. Juneja, "Meromorphic univalent functions with positive coefficients," Bull. Austral, Math. Soc. 32(1985), 161-176.

[5] S. Owa, "On the classes of univalent functions with negative coefficients," Math. Japon 27, No. $4(1982), 409-416$.

[6] S. Owa, "On the starlike functions of order $\alpha$ and type $\beta$," Math. Japon 27, No. 6(1982), 723-735.

[7] S. Owa, "On the Hadamard products of univalent functions," Tamkang J. Math 14(1983), 15-21.

Department of Mathematics, College of Science, University of Bahrain, P.O. Box 32038, ISA TWON, BAHRAIN. 